E-ISSN 2237-2660

\title{
Le Corps Performatif: les enjeux des scènes contemporaines
}

\author{
Sylvie Roques \\ Centre Edgar Morin (EHESS/CNRS) - Paris, France \\ Université d'Evry - Évry, France
}

RÉSUMÉ - Le Corps Performatif: les enjeux des scènes contemporaines - Cet article présente la mise en perspective d'une recherche d'une dizaine d'années, faisant croiser le regard sur le texte dramatique porteur de représentations corporelles, et l'examen de formes scéniques actuelles où la présence du corps est indéniable. S’il est impossible de limiter le théâtre au texte, notre position est qu'il est aussi impossible d'y échapper. La première partie met en perspective une analyse textuelle d'un corpus de textes contemporains européens révélant nombre de représentations corporelles. La seconde partie interroge la chair des mots car la parole est une matérialité tangible, porteuse d'une originalité particulière. La troisième partie est consacrée enfin aux formes performances émergeantes sur les scènes européennes où le corps acquiert toute sa densité et sa force.

Mots-clés: Sémiotique Théâtrale. Analyse Textuelle. Corps. Représentation. Performance.

ABSTRACT - Performative Body: the stakes in the contemporary stages - This article presents a research for decade making cross the look on the expanding dramatic text of physical representations and the examination of current scenic forms where the presence of the body is undeniable. If it is impossible to limit the theater to the text, our position is that it is also impossible to escape it. The first part puts in perspective a textual analysis of a corpus of revealing contemporary texts a number of physical representations. The second part questions the flesh of the words because the word is a tangible materiality, a carrier of a particular originality. The third part is dedicated to the forms emerging performances on the European stages where the body acquires all its density and its strength.

Keywords: Semiotics Theatrical. Textual Analysis. Body. Representation. Performance.

RESUMO - O Corpo Performativo: questóes da cena contemporânea - $O$ artigo coloca em perspectiva uma pesquisa de cerca de dez anos, cruzando um olhar sobre textos dramáticos que contêm representaçóes do corpo, e o estudo de formas cênicas atuais nas quais a presença do corpo é evidente. Considerando que é impossível limitar o teatro ao texto, afirmamos que também é impossível evitá-lo. A primeira parte do artigo coloca em perspectiva uma análise textual de um corpus de textos contemporâneos europeus que apresentam diversas representaçóes corporais. A segunda parte questiona o aspecto carnal das palavras, pois a fala é uma materialidade tangível, portadora de uma originalidade particular. A terceira parte trata das performances emergentes nas cenas europeias nas quais o corpo assume completamente sua densidade e sua força.

Palavras-chave: Semiótica Teatral. Análise Textual. Corpo. Representaçáo. Performance. 
D'emblée le théâtre est corps et c'est là même sa spécificité (Ubersfeld, 2001, p. 224). Il est aussi langage, représentation, accumulation de signes discursifs. Sur un plateau de théâtre, le texte est incarné par le corps de l'acteur et perçu par le spectateur dans sa totalité ou dans sa fragmentation. Or ce corps donné à voir est bien présent initialement dans le texte théâtral, en attente d'être incarné ou proféré. Mon propos sera de m'interroger sur la manière dont il peut être porté par le mot, autant que sur la manière dont il peut triompher malgré l'absence de mot. Cet article s'appuie sur un bilan de recherches universitaires effectuées depuis dix ans faisant croiser le regard sur le texte dramatique porteur de représentations corporelles et l'examen de formes scéniques actuelles où la présence du corps est indéniable.

De manière classique, en effet, la matérialité du théâtre réside en premier lieu dans le langage (phoné). C'est alors à son sens, à sa valeur de signe, que vont généralement les études portant sur l'acte théâtral, soulignant ses ressources d'évocation, son rôle dans le drame ou le récit. Cette mise en mots du corps mérite toute notre attention. Mais au-delà, le langage théâtral est sans doute d'avantage encore. Il est déjà, à travers la parole et sa déclamation, matérialité, puissance évocatrice, réalité de chair. On sait, en effet, d'après les travaux de Patrice Pavis, que le discours théâtral est analysable comme "[...] une performance discursive produite par un sujet énonciateur [...]" (Pavis, 1978, h1). Il devient alors texture sémiologique, émanation sonore et incarnée. Il atteint le spectateur dans son effet aussi physique qu'émotionnel. Ce qu'il convient de prendre en compte avec la plus grande des précisions. Un tel questionnement peut également s'inscrire aussi dans une mise à distance du texte conçu comme seule référence centrale de l'événement théâtral et perçu plutôt l'un des éléments du travail critique concernant la scène.

Reste pourtant une autre dimension encore de cette valeur de corps dans le théâtre: celle où le physique projette son sens hors de tout langage, hors de tout signe discursif. Gestes et expressions charnelles atteignent ici une puisssance spécifique. C'est le cas de nombre de tentatives performatives actuelles. J'entendrai ce terme de "performance", non pas au sens étroit comme relevant des arts plastiques mais davantage comme "forme performance” selon l'expression de Christian Biet (Biet, 2013, p. 23). Ce terme relève des distinctions proposées par Richard Schechner entre "being", suggérant l'existence d'un corps ou d'une chose et "doing" qui re- 
présente l'activité de cette chose et/ou de ce corps qui existent. La performance en ce sens est conçue comme "showing doing": "[...] ce qui fait que cette activité est soulignée, organisée, vue, privilégiée" (Biet, 2013, p. 24). De tels processus sont à l'œuvre dans les propositions scéniques ici étudiées et auxquelles nous avons assisté. L'action corporelle y est centrale. La performance, au sens large, réside alors dans la tentative de faire exister un sens issu de la seule chair, un caché, bousculer les modes trop communs de sentir et d'éprouver, ébranler tout conformisme dans le ressenti du corps. Elle magnifie quelque valeur exclusive du corps. Notre projet consiste à tenter de traverser les trois valeurs de sens suivantes et d'en montrer la force autant que la spécificité dans le théâtre : 1/ le langage comme signe, 2/ le langage devenu corps, $3 /$ le corps lui même devenu signe, unique et triomphant. Il s'agira surtout de montrer comment cette dernière catégorie, le corps signe (le fait qu'il manifeste une expression précise) doit être l'objet d'une prospection toute particulière sur les divers modes d'expression et les types de catégories d'action qu'elle mobilise.

\section{Le Corps mis en Mots}

Le texte dramatique préexiste le plus souvent à la représentation et aux ajustements du plateau. Autrement dit, le texte semble premier. Déjà Aristote indiquait que l'œuvre tragique peut s'accomplir dans la seule lecture et ne pas trouver comme unique issue celle d'un hypothétique devenir scénique. Elle peut se comprendre et se vivre par le texte seul. C'est bien encore ce que propose Michel Corvin lorsqu'il évoque l'idée d'une "[...] lisibilité projective du texte de théâtre" (Corvin, 2002, p. 203). Une telle lisibilité existe au sens strict du terme dont on ne peut faire l'économie et implique comme prérequis la lecture du texte avant d'en envisager la représentation: "Autrement dit un texte de théâtre ne serait pas à jauger en référence à quelque dramaturgie que ce soit, mais au trébuchet. - d'abord - de la longue et patiente dégustation d'un lecteur, installé dans son espace mental et sa temporalité libre. Foin des modèles de représentations" (Corvin, 2002, p. 204). Le texte, dans ce cas, domine et pré-existe au spectacle qui en sera donné. Plus précisément, il convient de distinguer un " texte premier " que constitue le "texte à dire" ou à lire qui diffère d'un texte "second", correspondant ici à son actualisation scénique, résultant du travail au plate- 
au (Biet; Triau, 2006, p. 546), celui que l'expérience du plateau aura modifié pour l'enrichir et l'ajuster.

En revanche, l'éloignement du théâtre à l'égard du seul domaine de la littérature a conduit au refus de traiter le texte de théâtre comme un texte littéraire et a contribué à ce désintéressement depuis une cinquantaine d'années, focalisant les recherches sur la spécificité de l'événement théâtral et de sa mise en scène (Pavis, 2001, L1). Une complexité demeure, quoi qu'il en soit, mise en évidence par Fernando de Toro (Toro, 2008, p. 109). Le problème fondamental étant que le théâtre ne se limite pas soit au texte soit à la mise en scène. Il importe de dépasser cette dichotomie et de ne pas faire l'impasse sur le contexte social et culturel dans lequel il s'inscrit.

S’il est impossible de limiter le théâtre au texte, notre position est qu'il est aussi impossible d'y échapper. Texte troué, en attente de représentation et objet culturel, le texte théâtral peut en ce sens témoigner aussi d'une certaine vision $d u$ monde et se percevoir comme susceptible d'explorer le corps en langue: il en est l'énonciation. Choisir de s'intéresser aux représentations spécifiquement textuelles du corps dans la littérature contemporaine peut sembler relever de préoccupations toutes actuelles. Il reste qu'une telle entreprise soulève de nombreux questionnements. L'abord des représentations du corps dans la littérature dramatique renvoie généralement à des registres très différents depuis le texte jusqu'à la dramaturgie et ses choix. Le mot, pour sa part, n'en est pas moins porteur de sens autant que de poiésis et de chair. Cette mise en mots du corps dans l'œuvre théâtrale mérite notre attention. L'étude d'un corpus de quarante-quatre pièces de théâtre contemporain européen fait apparaître un spectre, tout à fait caractéristique de la culture corporelle. Mon propre projet était alors d'appréhender à travers les énoncés des personnages leur trace linguistique, envisagée comme reflétant l'acte d'énonciation qui le supporte, tout comme reflétant un sens plus générique porteur de culture et de sensibilité. C'est dans ces traces de langage mêmes, que peuvent être cherchées les représentations du corps. D’où le parti pris d'une sémiotique s'appuyant sur les base d'une analyse du discours mis en œuvre dans les pièces étudiées.

Les résultats obtenus au terme de l'analyse textuelle ont mis à jour toute une typologie de représentations du corps (Roques, 2011). Le vocabulaire et les items recensés ont révélé à quel point la sensibilité à l'égard du corps était présente. De grandes catégories d'images ont pu être répertoriées: 
d'une part, celles qui suggèrent la représentation d'un corps blessé lié au contexte de la guerre, d'un corps sensible à la douleur ou à la maladie, d'un corps non organique qui s'apparente à un corps glorieux et d'autre part, celles qui indiquent un corps soumis aux pouvoirs socio-économiques. Corps violenté, corps sensible, corps glorieux, corps dépendant. II s'agit bien de quatre versants différents, de manières d'être et de représentations. Le discours théâtral serait ici à mettre en correspondance avec notre temps. Le corps violenté, évoqué dans les textes dramatiques étudiés, désigne sans ambigïté la sensibilité actuelle avide de sécurité, inquiète de toute blessure, de toute agressivité, forgeant mille défenses pour mieux se protéger. Reviennent régulièrement ici les mots de guerre, ennemi, crier, hä̈r, les frontières corporelles étant dès lors mises à mal. La pièce de Philippe Minyana ${ }^{1}$, Les guerriers (1988), est d'ailleurs une des plus représentatives à cet égard.

Le corps sensible constitue un autre thème. Il se donne comme une quasi réponse au mal par ailleurs éprouvé, rempart quasi personnalisé dans nos sociétés individualistes. Il est celui dont les auteurs citent avec insistance toutes les composantes, suggérant leur valeur, leur manière possible d'incarner force et affirmation: la bouche, le corps, le visage, la peau, la respiration, le muscle. Autant de parties faites pour résister et pour affirmer. Corps inquiétant encore parce que fragile et précieux, vulnérable même, dont sont quelquefois citées les parties intérieures, les dessous comme autant de lieux obscurs et perturbants.

Le corps glorieux est caractérisé par un univers lexical fortement différent des deux précédents. Il apparaît non plus fragmentaire, mais magnifié. Si important même qu'il semble dans le texte lui-même se ramasser tout entier dans le souffle des mots. C'est ce que semble dire Valère Novarina ${ }^{2}$ : "[...] notre esprit qui êtes, dites si nous sommes! [...] Nous voudrions nous aussi maintenant nous dépouiller des mots en parlant. Au secours, éternel, sois présent à ma place! J'y arrive pas!” (Novarina, 1997, p. 105-106). Les items parole, matière, gloire, "néant" sont représentatifs de cette thématique. Les représentations du corps qui en émergent paraissent explorer la négation et le vide pour mieux atteindre un corps glorieux devenu de part en part un corps spiritualisé. Corps si présent que son instantanéité correspond quelquefois à son effacement. Le corps dominé donne le sentiment qu'existent des forces, dont les effets sont physiques, contraignant et soumettant chacun d'entre nous. Un tel corps s'inscrit dans l'espace réglementé 
du travail, suggérant une vision implacable d'un monde économique où se laissent quelquefois deviner la catastrophe et l'auto-dévoration. Les processus impitoyables de la concurrence et de la recherche du profit peuvent y atteindre leur paroxysme. S'y ajoute la sensation d'étouffement et de contrôle sur les corps.

Je m'attacherai plus spécifiquement à saisir deux types de représentations du corps émergeant de cette analyse textuelle et ayant quelques résonnances communes: ceux relevant de pièces de deux auteurs contemporains que sont Philippe Minyana et Valère Novarina, deux auteurs très médiatisés depuis les années 1990 et qui figuraient dans le corpus étudié et se détachent de l'analyse textuelle. Le choix de ces deux auteurs me semblait d'autant plus pertinent qu'ils paraissaient particulièrement révélateurs d'un courant marquant aujourd'hui donnant une place primordiale à la parole et à sa profération: celui d'un théâtre de paroles. Rapprochement d'autant plus important enfin que demeure indéniable leur fort intérêt pour le corps. Rappel aussi d'un incontournable réalisme: le corps est d'abord matière. Il peut se vivre sous cette forme.

Les représentations d'un corps de type organique semblaient communes chez ces deux auteurs. Chez Novarina, les références anatomiques ou physiologiques retiennent tout particulièrement l'attention, notammemnt dans la pièce Lettre aux acteurs (Novarina, 1989, p. 22)

Le corps en jeu n'est pas un corps qui exagère (ses gestes, ses mimiques), l'acteur n'est pas un 'comédien', pas un agité. Le jeu, c'est pas une agitation en plus des muscles sous la peau, une gesticulation de surface, une triple activité des parties visibles et expressives du corps (amplifier les grimaces, rouler des yeux, parler plus haut et plus rythmé.

Elles induisent une géographie du corps mêlant organes et orifices, humeurs et muscles, comme si l'auteur avait voulu épuiser toutes les possibilités du corps humain et instaurer une nouvelle circulation. Ce corps de type organique qui révèle son intérieur est aussi repérable chez Minyana. Cependant chez cet auteur si l'organicité n'est pas oubliée il se mêle aussi au fantastique et à l'imaginaire. Sang, odeurs, sécrétions corporelles semblent acquérir leur propre autonomie et échapper à la simple volonté du personnage comme dans Volcan ou Les Guerriers. 


\section{La Chair des Mots}

L'intérêt de ces derniers textes est pourtant d'évoquer davantage. À cette mise en mots du corps ainsi explicitée s'ajoute une nécessaire prise en compte d'une plus grande complexité: celle d'une mise en corps du mot luimême, à travers le triomphe de la parole. C'est que la parole, ainsi comprise est plus complexe que les mots. Elle a une force. Elle se fait chair. Cette matérialité est tangible chez Valère Novarina: "Notre chair physique c'est la terre mais notre chair spirituelle la parole: elle est l'étoffe, la texture, le tissu, la matière de notre esprit" (Novarina, 1999, p. 16). Derrida le montre d'ailleurs à sa manière: l'oralité est porteuse d'une originalité toute particulière, laquelle ne saurait réduire la parole à l'écriture. Cette parole est chair, ce qui lui donne une résonance spécifique. Elle est matière, tonalité, tremblement, émotion. Derrida le dit dans sa formulation apparemment complexe, alors que celle-ci, une fois ce principe reconnu, est limpide: «Le privilège reconnu non seulement au langage mais au langage parlé (voix, écoute, etc...) consonne avec le motif de la présence comme présence à soi " (Derrida, 1972, p. 160). Il faut ajouter que cette transcription ne saurait épuiser l'originalité intrinsèque de la parole.

D'autant qu'il ne faut pas oublier que le texte théâtral n'est pas seulement lu mais il est parlé, il est aussi situé, ce qui lui donne inévitablement un versant particulier. Comme s'interroge Michel Corvin: "Comment, alors, peut-on voir les mots?”. Une réponse est apportée: cette opération devient possible si l'on transforme les mots "[...] en éléments sensibles, c'est àdire en voix et en corps mouvants" (Corvin, 2008, p. 126). Michel Corvin ajoute encore: "[...] ce que, à la suite de Novarina, on pourrait appeler 'la théâtralité respiratoire de la page"” (Corvin, 2008, p. 126). La voix prend alors le relais rendant tangibles pensées et affects. Elle apporte une sorte de traduction métaphorique de ce qui se trouve généralement au centre du théâtre occidental à savoir l'exposition d'un conflit interne ou externe. La parole se fait alors véritable action. Des artistes tels Vincent Barras (2013) ${ }^{3}$ l'ont bien compris et ont utilisé un tel processus, s'appuyant sur des formes de performances verbales, les poussant jusqu'au paroxysme. Une exploration systématique du corps depuis ses bruits singuliers, ses bégaiements, et autres lapsus ou parasites peut par exemple en être proposée. 
Cette fonction du logos comme praxis n'est pas neuve, une telle vision atteste d'un héritage. Elle a été déjà mise en évidence notamment par Roland Barthes lorsqu'il évoquait Phèdre, la tragédie de Racine:

Dire ou ne pas dire? Telle est la question. c'est ici l'être même de la parole qui est porté sur le théâtre: la plus profonde des tragédies raciniennes est aussi la plus formelle; car l'enjeu tragique est ici moins le sens de la parole que son apparition, beaucoup moins l'amour de Phèdre que son aveu. Ou plus exactement encore: la nomination du Mal l'épuise tout entier, le Mal est une tautologie, Phèdre est une tragédie nominaliste (Barthes, 1963, p. 115).

Parler c'est agir avait déjà souligné l'abbé d'Aubignac au XVII siècle et l'action scénique traduite en paroles, est alors "[...] la métaphore d'une action qu'on ne verra pas" (Corvin, 2008, p. 126). Passer des mots, à leur profération, leur incarnation et à leur action sur un plateau de théâtre, tel a été le renversement de paradigme illustrant ma recherche.

\section{D'un Corps agi à un Corps sans Mots}

Si de nos jours le texte a perdu sa prééminence, l'attention au corps a grandi, comme s'est déplacé le statut d'un artiste devenu acteur-performer et celui du jeu. L'artiste se fait performer car le rôle supposé incarné est mis à distance et l'acteur devient celui qui, "[...] dans un temps éphémère et en un lieu particulier, agit et parle, chante et danse, ostensiblement et en son nom propre" (Biet, 2013, p. 25). Aussi, c'est lui-même qu'il met en scène de manière tangible. Cette mise à distance critique de l'acteur accompagne d'autres bouleversements. Plusieurs études récentes, le soulignent: le théâtre occidental conçu comme lieu de représentation est mis en crise (Bougnoux, 2006, p. 8). Hans-Ties Lehmann a pu appeler un "théâtre postdramatique" (Lehmann, 2002), marquant ainsi ce qu'il juge être une rupture. Il importe de préciser le changement de paradigme mis en place. Le dramatique n'y est pas caduc mais apparaît plutôt comme déplacé. Un renversement est alors perceptible "l'hégémonie du drame sur le théâtre" est suspendu (Guenoun, 2011, p. 651). La représentation émancipée évoquée par Bernard Dord semble advenir, le texte devenu un simple élément du spectacle. Le corps participe à ce renouvellement, mettant en ouvre des effets de présence (Féral, 2011) sidérants et sans distance. Ces effets sont liés à la qualité de présence de l'acteur (Féral, 2011). Un versant central de la performance théâtrale est bi- 
en là: exprimer le tout dans un immédiat sans distance, donner à la présence de chair, à sa densité la plus tangible, la valeur d'un dire sans parole. Dans cette perspective, il s'en suit un brouillage possible de frontières au niveau de la réception. La mise à distance qu'opérait la catharsis s'en trouve affaiblie. C'est bien ce processus qu'il s'agit de repérer sur les scènes contemporaines dans un certain nombre de propositions scéniques.

Longtemps cette émergence intempestive du corps a servi une contestation par gestes et pulsions dans les années 1960-1970. Elle prenait place dans des performances ou des happenings qui participaient "[...] d'un mouvement de contestation des valeurs traditionnellement attachées à l'art et se voulait l'éperon d'un mouvement de rejet de l'œuvre artistique comme objet" (Féral, 2011, p. 222). Si de telles performances pouvaient avoir un rôle fondamental de transgression et occuper une fonction particulière dans la critique et la dénonciation à l'encontre de la société et de l'oppression exercée sur l'individu, de nos jours un déplacement s'opère. Les spectacles de forme performative conçus comme présentations et non plus représentations s'accompagnent sans doute d'un recul de l'impact critique envers la société, et ce, malgré toutes les formes ludiques et nouvelles qui ont pu s'inventer. Elles montrent autre chose et sont à cet égard décisives. Une prééminence du sujet s'y exprime et s'accompagne d'une insistance sur ce qu'il éprouve le plus charnellement. D'où cette dominance de longues phases scéniques consacrées aux apparences et accidents de la peau, aux contacts et frôlements des corps, à leur malaxage, à leurs manifestations organiques comme dans la pièce Et Balancez mes cendres sur Mickey (2006) où les acteurs luisants de miel figurent de "magnifiques tableaux de la peinture flamande" (Tackels, 2016, p. 15). De nouvelles formes pourtant sont inventées faisant place au collectif tout autant qu'au sujet. Le théâtre de Rodrigo Garcia ${ }^{4}$ en est un bon exemple. C'est qu'il renoue avec des pratiques de contestation, mais révisées, repensées, tout en donnant une place marquante au performer. Il s'inscrit ainsi dans les traces d'un Peter Handke (Handke, 1993), et s'inspire de sa posture d'interpellation en proposant bon nombre de monologues plus ou moins outrageants - spectacles qui participent aussi à la déconstruction appliquée de l'illusion théâtrale et de ses avatars. Tous les piliers de ce qui constitue le drame occidental y sont pulvérisés. Ascension de l'individu sans doute, mais revendication collective encore, ce qui est original. La contestation d'ailleurs a aussi elle-même changé. La stigmatisation 
ne vise plus les vieilles aliénations contestées dans le passé, mais la standardisation imposée par la consommation, l'uniformisation venue du gadget, l'obsession du sexe aussi, un vide, aux yeux de Rodrigo Garcia, fait d'un trop inutile et superficiel. L'originalité de ce dernier artiste est de dépasser les vieux modèles contestataires des années 1960, centrés sur la remise en cause des interdits et de l'autorité, pour s'attacher à stigmatiser une société de consommation annihilant les individus par une culture du plus qui ne devient rien d'autre qu'une culture du vide. Chacune de ses scènes, chacune de ces pièces est une déconstruction obstinée du trop.

Finalement tout concourt dans les spectacles de Rodrigo Garcia à nous mettre en état d'immersion: assaillis de tous côtés par les symboles de la consommation de masse, la nourriture qui sort de ses gonds, un écran sursaturé par un déferlement d'images hypnotiques ou transgressives. Ce qui permet précisément de donner intensément place au corps. Ainsi dans l'un de ses derniers spectacles, qui a fait polémique à cause des références antireligieuses, Golgota Picnic (2011), le plateau est envahi de petits pains et une sorte de pique- nique improvisé s'y organise, alors qu'une caméra placée au centre filme en gros plan les acteurs, objet d'infinies discussions. Pas de fiction ni de personnages mais l'incorporation d'actions de types performatives est repérable. J'en reprendrai quelques-unes: un des acteurs-performers avale un hamburger qu'il vient de se confectionner et le dégurgite immédiatement après chaque bouchée. La caméra scrute et nous livre en gros plan sa régurgitation. Une autre séquence tout aussi déstabilisante a lieu plus tard lorsque les acteurs-performers se dénudent et effectuent des pyramides improbables, utilisant tout autant leurs jambes et bras que leurs cheveux ou leurs poils. De même une séance de Body-painting a lieu: de la peinture bleue est utilisée, sans doute en référence aux anthropométries d'Yves Klein. Se mélangent alors couleur et sécrétions corporelles. Théatre sans doute, mais largement infiltré par le faire au détriment du discours, largement centré sur le spontané aussi plus que sur le récit, centré encore sur le choc physique, le dispositif spatial, plus que sur toute rhétorique ou tout discours. Ce sont de telles scènes extrêmes, largement immergées dans l'investissement charnel, qui me paraissent caractériser une voie marquante du théâtre contemporain.

Ma réflexion s'est aussi orientée vers des dispositifs mis en place pour une durée restreinte dans les spectacles les plus variés, évènements courts, 
effectués en présence de spectateurs, avec leur présentation dénuée souvent de récit, de trame dramatisée, alors même que leur cadre, leur plateau, semble lui-même théatralisé. De tels dispositifs gagnent en importance, formules hybrides où les corps s'expriment, le spectacle se donne, la scène est présente, sans qu'existe un dialogue ou une quelconque explicitation discursive.

Ainsi les pratiques esthétiques et les expérimentations artistiques de Yann Marussich 5 inquiètent la perception du spectateur en instaurant avec lui un rapport particulier qui échappe à la catégorisation illusion et/ ou distanciation. Elles travaillent à donner généralement à percevoir ce que le spectateur ne perçoit pas habituellement, en détournant les codes des arts scéniques. Plusieurs de ses performances sont à cet égard des plus caractéristiques.

Dans l'installation performative intitulée Bleu Remix (2007), le spectateur s'approche au plus près du performer, se collant à la vitre pour regarder Yann Marussich se mettre à transpirer en bleu dans sa boîte en plexiglass. Les effets sont tangibles: la distance critique est réduite et les effets massifs de présence produisent la captation visuelle. Le corps du performer y semble gagner en densité et en force. Grâce au dispositif visuel et sonore mis en place, la perception bouscule les cadres habituels de la réception comme l'espace et le temps, et joue sur les paradoxes entre le proche et le lointain, les effets de captation voire de sidération et de distance.

Le corps y est central dans sa dimension la plus réelle. Des effets de présence en émergent, lié à la proximité de l'artiste et provoquent l'empathie. Dans ce même mouvement du proche et du lointain, une distance des plus ténue demeure: la fragile séparation entre l'artiste et le spectateur fait écran et se concrétise par la paroi des boîtes où il est installé, par le dispositif où il s'insère à titre individuel.

Dans Autoportrait dans une fourmilière (2003) les spectateurs circulent autour du cercueil en verre de Marussich où celui-ci est allongé immobile au milieu des fourmis. Le dispositif en place fait intervenir des caméras pour scruter au plus près l'immobilité de l'artiste au milieu de l'agitation des fourmis et des casques mis à la disposition des spectateurs leur permettent d'entendre une bande son. Ceux-ci semblent accompagner le performer dont l'immobilité est frappante et ressemble à celle d'un mort (Marussich, 
2013). Aux mouvements de circulation des publics s'ajoutent murmures et paroles ou chansons illustrant leur compassion, leur critique ou leur humour. Alors même que l'immobilité fermement adoptée trouble d'autant plus le spectateur que la souffrance du performer peut fortement s'imaginer sinon se voir.

Dans Traversée (2004), le dispositif s'apparente à une torture: un câble entoure le cou de Yann Marussich. C'est bien le seul corps du performer dans sa nudité extrême qui est offert au regard critique du spectateur, sans distance. L'artiste est soumis au bon vouloir des spectateurs prenant parti et choisissant soit de resserrer l'étau soit de l'en dégager. S'expriment alors les luttes conscientes ou inconscientes, les pulsions et les passions les plus inavouées qui semblent animer les publics. Le spectacle se fait interactif et gagne la salle. Le regard n'est plus seulement focalisé sur l'artiste mis à distance mais s'élargit à ceux qui l'entourent et y participent, défragmentant l'attention, la démultipliant à tout un jeu animant les consciences.

Dans de tels exemples, le corps performatif introduit des effets de réels. $\mathrm{Au}$ centre du plateau, il en détourne les usages et les conventions. Des tendances s'avèrent repérables: travailler sur la perception, l'immersion et la déconstruction. Un certain nombre d'expériences artistiques font de l'expérience perceptive, de son bouleversement et de son activation un enjeu essentiel. Ce qui s'avère important dans des périodes, comme les nôtres, où les certitudes habituelles semblent remises en cause et où s'estompe le sentiment de pouvoir tout dominer, alors même que s'accroît la nécessité de s'interroger, sinon de s'inquiéter.

Pour conclure, la spécificité de certains textes dramatiques s'inscrivant dans un théâtre de parole spécifique à une période déterminée en Europe a été mise à jour. Nombre de représentations corporelles particulières en émergent. Or ce qui peut s'entendre dans le dire des personnages, tels ceux proposés par Novarina, excède le simple système communicationnel habituel. Le langage se fait chair, il devient corps. Cette émergence du corps au delà des mots se fait encore plus tangible si l'on se situe du côté des scènes contemporaines. Un paradoxe ne peut manquer d'apparaitre, soulignant les limites et les frontières d'un corps donné à voir, exhibé dans sa réalité la plus concrète et sa matérialité physiologique, placé désormais au cœur du drame. 


\section{Notes}

1 Philippe Minyana est l'auteur d'une trentaine de pièces montées par des metteurs en scène comme Edith Scob, Catherine Hiégel, Alain Françon. Il a exercé le métier d'acteur et de directeur d'acteurs. Il a été auteur associé au Théâtre Ouvert et au centre dramatique de Dijon dirigé alors par Robert Cantarella. Il illustre "[...] la tendance propre au théâtre européen des années 1980, du travail sur la mémoire et du récit de vie" cf. Philippe Minyana (Abirached, 2011, p. 680).

2 Valère Novarina est écrivain, peintre et photographe. Il aborde l'écriture dramatique au début des années 1970. Ses pièces sont montées par Jean-Pierre Sarrazac, Marcel Maréchal. André Marcon remporte un grand succès en 1986 au Bouffes du Nord avec la pièce de Novarina Le Discours aux animaux. L'auteur mettra en scène ensuite ses propres textes. Cf. Valère Novarina (Abirached, 2011, p. 683).

3 Vincent Barras est artiste performer et Professeur d'histoire de la médecine, à l'Institut universitaire d'histoire de la médecine et de la santé publique, à la faculté de biologie et de médecine de l'Université de Lausanne et CHU Vaudois.

4 Rodrigo Garcia est auteur, metteur en scène espagnol. Ses pièces sont fréquemment montées sur les scènes françaises et dans les festivals.

5 Yann Marussich est artiste performer, chorégraphe et réside à Genève.

\section{Références}

ABIRACHED, Robert (Ed.). Anthologie de l'Avant-Scène Théâtre Le Théâtre Français du XXe Siècle. Paris: l'avant scène théâtre, 2011.

BARRAS, Vincent. Parole performée. Revue Communications [Performance], Paris, Le Seuil, n. 92, p. 253-261, 2013.

BARTHES, Roland. Sur Racine. Paris: Le Seuil, 1963.

BIET, Christian. Pour une extension du domaine de la performance (XVII ${ }^{e}-X{ }^{e}$ siècle). Revue Communications, Paris, Le Seuil, n. 92, p. 21-35, 2013.

BIET, Christian; TRIAU, Christophe. Qu'est ce que le Théâtre?. Paris: Gallimard, 2006.

BOUGNOUX, Daniel. La Crise de la Représentation. Paris: La Découverte, 2006. 
CORVIN, Michel. L'Écriture Théâtrale contemporaine entre modèles de représentation et illisibilité. Études Théâtrales [Ecritures dramatiques contemporaines [1980-2000], Louvain, n. 24-25, p. 202-204, 2002.

CORVIN, Michel. Pour une réception musicale du théâtre contemporain. Revue Communications [Théâtres d'aujourd'hui], n. 83, p. 123-130, 2008.

DERRIDA, Jacques. Marges, Paris. Editions de Minuit, 1972.

FÉRAL, Josette. Théorie et Pratique du Théâtre. Au-delà des limites. Montpellier: l'Entretemps, 2011.

GUENOUN, Denis. La représentation en débat. In: ABIRACHED, Robert (Ed.). Le Théâtre Français du XX ${ }^{\mathrm{e}}$ Siècle. Paris: 2011. P. 649-657.

HANDKE, Peter. Outrage au Public et Autres Pièces Parlées (Prédiction, Introspection, Appel au secours) (1964-1965). Traduites par Jean Sigrid. Paris: L'Arche, 1993.

LEHMANN, Hans-Thies. Le Théâtre Post-Dramatique. Traduites par Philippe-Henri Ledru. Paris: L'Arche, 2002.

MARUSSICH, Yann. Voyage(s) dans l'Immobilité. Revue Communications, Paris, Le Seuil, n. 92, p. 239-251, 2013.

NOVARINA, Valère. Le Théâtre des Paroles. Paris: P.O.L., 1989.

NOVARINA, Valère. L’Espace Furieux. Paris: P.O.L., 1997.

NOVARINA, Valère. Devant la Parole. Paris: P.O.L., 1999.

PAVIS, Patrice. Remarques sur le discours théâtral. Degrés [Théâtre et sémiologie], Bruxelles, v. 13, h1-h10, 1978.

PAVIS, Patrice. Thèses pour l'Analyse du Texte Dramatique. Degrés, Bruxelles, n. 107108, L1, 2001.

ROQUES, Sylvie. Le corps mis en mots dans le théâtre contemporain. Sémêion, Paris, Editions Universitaires de la Sorbonne, n. 8, p. 63-70, sept. 2011.

TACKELS, Bruno. Notes de cuisine/Notes de salle. Théâtre Public, Montreuil, n. 220, p. 10-15, avril-mai 2016.

TORO, Fernando de. The end of theatre semiotics? A symptom of an espitemological shift. Semiotica, 168-1/4, p. 109-128, 2008.

UBERSFELD, Anne. Lire le Théâtre. Paris: Belin, 2001. 
Sylvie Roques est chercheure associe au Centre Edgar Morin (Centre National de la Recherche Scientifique/Ecole des Hautes Etudes en Sciences Sociales) depuis 2006. Après avoir soutenu une thèse en Esthétiques Sciences et Technologies des Arts option théâtre en 2005 à l'Université de Paris 8 consacrée au corps et ses images dans l'écriture dramatique contemporaine, ses recherches ses sont orientées vers le théâtre post-dramatique et le théâtre performatif. Elle a soutenue en novembre 2014 une habilitation à diriger des recherches à l'Université de Paris 10, ayant pour titre Auctorialité et spectacularité XIX-XXe siècles.

E-mail: sroques@noos.fr

Ce texte inédit, révisé par Annelyse Gayraud, est également publié en portugais dans ce numéro.

Reçu le 31 Mars 2016 Accepté le 5 Septembre 2016 